\title{
High prevalence of antimicrobial resistance among Gram-negative bacteria isolated from poultry
}

\author{
Abdelraouf A. Elmanama', Mariam R. Al-Reefi', \\ Mohammed A. Albayoumi², Alaa M. Marouf ${ }^{1}$, \\ Islam F. Hassona ${ }^{3}$
}

\section{Abstract}

Background: Infection with Multidrug resistant bacteria (MDR), such as Escherichia coli and Salmonella spp. are considered as threat to the human health care system. In recent years, these MDR bacteria have been found increasingly inside and outside the hospital environment. Food animals (meat and poultry) are increasingly colonized with MDR bacteria, thus posing an additional concern. This study is intended to determine susceptibility and resistance pattern of pathogenic Gramnegative bacteria isolated from rectal swabs of chicken against16 antibiotics.

Methods: A total of 216 cloacal swab samples from Gaza strip poultry farms and 87 (frozen and fresh meat samples obtained from slaughter houses and retails) were collected over the period from June 2017 to June 2018. Isolation and identification of organisms were achieved using standard bacteriological techniques. Antimicrobial susceptibility test was performed according to standard protocols.

Results: A total 360 Enterobacteriaceae isolates, and 56 Gram-negative non fermenter were recovered. The predominant Enterobacteriaceae isolate was Citrobacter spp. (22.6\%), followed by Enterobacter spp. (17.6\%) and E. coli (16.5\%). High rates of resistance against ampicillin (85.4\%) and trimethoprim/sulfamethoxazole (80.1\%) followed by chloramphenicol (74\%) were recorded. Few samples were positive for Salmonella spp. and Shigella spp. Of the tested Enterobacteriacae isolates, $94.7 \%$ were multidrug resistant (MDR), and $31.4 \%$ of non-fermenting bacilli (NFB) were MDR. Carbapenem resistance was found to be high among isolates; $51.9 \%$ for imipenem and 1.8\% for meropenem.
1 Medical Laboratory Sciences Department, Faculty of Health Sciences, Islamic University of Gaza, P.0 Box 108, Gaza, Gaza strip, Palestine.

2 Veterinary Services, Ministry of Agriculture, Gaza, Palestine.

3 Al-Shifa Hospital, Ministry of Health, Gaza, Gaza strip, Palestine.

Contact information:

Abdelraouf A. Elmanama.

झ elmanama_144@yahoo.com 
Conclusion: This study suggests that presence of high rates of MDR bacteria isolates in chickens constitutes an important reservoir of antimicrobial resistance in Gaza-Palestine which is a major public health concern.

\section{Keywords}

Multi-Drug Resistance; Antimicrobial Resistance; Poultry; Gaza- Palestine.

\section{Background}

All forms of life clearly play an important role within the context of the One World, One Health according to the concept of World Health Organization. Food animals can serve as sources for infectious diseases for humans resulting from direct contact or environmental contamination. In addition, food animals can also transmit pathogens to other animal species directly and indirectly through environmental dispersal [1].

Escherichia coli is one of the most common intestinal commensal organisms found in human and many animals and therefore it is widely disseminated in the environment. Investigating potential pathogenic $E$. coli strains and/or antibiotic resistance may provide information regarding human activity in select ecological niches [2]. Bacteria may acquire resistance to antibiotics under selective pressure, but they may also become resistant to antibiotic without direct exposure to an antibiotic through horizontally mobile elements including conjugative plasmids, integrons and transposons [3]. These genetic elements can frequently transfer antibiotic resistance genes from one bacterium to another through the intestinal tract of human and animals [4].

Monitoring the prevalence of resistance among indicator bacteria such as E. coli and enterococci in different populations such as animals, patients and healthy peoples, makes it feasible to compare the prevalence of resistance and to detect the transfer of resistant bacteria or resistance genes from animals to humans and vice versa [5]. However, few reports about the prevalence rates of antimicrobial resistance in $E$. coli and enterococci in food animals have been published [6].

In several countries, antibiotics such as peniciIlin, erythromycin and tetracycline are approved for growth promotion as well as therapeutic use in animals. Many of the antibiotics that are given to animals are closely related to medically important human drugs. Thus it is possible that the indiscriminate use of antibiotics of non-human use can lead to the development of resistance which could then be passed to human pathogens [7].

In Gaza strip, clinical studies have shown high and alarming rates of resistance [8-10]. Only one study has reported occurrence of colistin resistance (16\%) among E. coli from poultry which will be published soon. Data concerning resistance among isolates from food animals are scarce.

This study was performed with the aim of investigation the occurrence of antimicrobial resistance among Gram-negative bacteria recovered from poultry in Gaza strip. 


\section{Material and Methods}

\section{Samples collection and transportation}

The present research was conducted during the period from June 2017 to June 2018. A total of 216 cloacal swabswere collected from broiler chickens (two swabs from each chicken; one for Salmonella and Shigella isolation and the second for other Gramnegative isolation) and 87 meat samples (100grams each) were collected from broiler slaughterhouses and shops as fresh and frozen meat. Samples were obtained from different locations in Gaza (Rafah, Khan Younis and the Middle governorates). Samples were collected aseptically, kept in an icebox and transferred to the Microbiology Laboratory at the Islamic Universityof Gaza.

\section{Microbiological investigation Meat samples}

A $25 \mathrm{~g}$ meat sample was homogenized at low speed in a sterile blender with $225 \mathrm{ml}$ peptone water $(0.1$ $\%)$ for 2 minutes. This constituted the $10^{-1}$ dilution. MacConkey agar and Violet Red Bile Agar(VRBA) plates (for gram negative bacteria isolation) were streaked by a loopful of the $10^{-1}$ meat dilution. Well isolated bacterial colonies from both plates were picked and purified by streaking onto fresh media. The isolates were then identified by colony morphology and growth characteristics.

\section{Cloacal swabs}

One cloacal swabwas inoculated onto pre-labeled Blood agar, MacConkey agar, and VRBA plates. The inoculated plates were incubated at $37 \pm 0.5^{\circ} \mathrm{C}$ for 24 hours. The other swab was used to inoculate a tube of Selenite-F broth for Salmonella and Shigella isolation.

\section{Isolation of Salmonella and Shigella spp.}

A $0.1 \mathrm{ml}$ from the pre-incubated samples in peptone water and selenite $\mathrm{F}$ broth was streaked onto (Xylose Lysine Deoxycholate agar \& Salmonella Shigella agar) and incubated at $37^{\circ} \mathrm{C}$ for $24 \mathrm{hr}$. Following incubation, lactose non fermenting colonies with or without black centers were streaked onto urea spot test. Typical Salmonella and Shigella colonies on XLD and SSA that are negative urease test were subcultured into triple sugar iron agar slant and incubated for $24 \mathrm{hr}$ at $37^{\circ} \mathrm{C}$ then identified using API 20E (bioMerieux, France).

\section{Isolates identification}

Confirmed gram negative isolate were identified biochemically using API 20E kit. Gram stain, growth on MacConkey, Triple sugar iron agar and oxidase tests were also used according to Krumperman [11].

\section{Antibiotic Susceptibility Tests}

The disc diffusion test was done for all isolates based on Clinical Laboratory Standard Institute guidelines [12]. Commercial Antimicrobial impregnated discs were dispensed on the surface of cultures of MullerHinton agar and incubated at $35^{\circ} \mathrm{C}$ for $20 \mathrm{hrs}$. The diameters of the zones of inhibition were recorded to the nearest $\mathrm{mm}$ and classified as resistant, intermediate, or susceptible according to established interpretive chart [12].

\section{The Multiple Antibiotic Resistance (MAR) index} MAR (Multiple antibiotic resistance) index provides useful information for the evaluation of a health risks [13]. MAR index value higher than 0.2 is regarded as MDR. An isolate was regarded as MDR if they were resistant to one or more antibiotics of three different classes

\section{Statistical Analysis}

The data were analyzed with Statistical Package for the Social Sciences (SPSS), version 22.0 software (Microsoft SSPS, USA). 


\section{Results}

A total of 360 Enterobacteriacae, and 56 non-fermenting bacilli (NFB) were isolated from the three sampling locations (Table 1).

The results of the identification of isolates showed that the predominant isolate among Enterobacteriacae, was Citrobacter spp. (22.6\%), followed by Enterobacter spp (17.6\%) and E. coli (16.5\%) (Figure 1).

In general, amikacin and meropenem showed the highest activity among all the tested antimicrobial, while tetracycline, ampicillin and trimethoprim/sulfamethoxazole were the least active drugs as show in Table 2.

Table 3. Shows the result of the percentage resistance of the Enterobacteriacae isolates to each of the tested antimicrobials. A total of 416 isolates were tested against sixteen commercially available antibiotics. Among the tested isolates, none of the Enterobacteriacae and NFB were susceptible to all antibiotics. The majority of the Enterobacteriacae and Gram-negative non-fermenter isolates showed variation in their susceptibilities to different antimicrobials. Among Enterobacteriacae, resistance to amikacin is $0.3 \%$ and high rates of resistance were observed against tetracycline (94.1\%). High rates of resistance against ampicillin (85.4\%) and trimethoprim/sulfamethoxazole (80.1\%), followed by chloramphenicol (74\%) as shown in Table 2.

In general, almost all isolates showed high percentage of resistance to tetracycline, ampicillin, and trimethoprim/sulfamethoxazole. Klebsiella spp., Citrobacter spp., Morganella spp., Salmonella spp., and Shigella spp. were the only species that showed absolute susceptibility to meropenem. This pattern was so different with imipenem, wherein, almost all isolates exhibited resistance with the notable exception of Shigella spp. (Table 3).

Table 4 shows the lowest resistance rates among NFB isolates to azithromycin (0\%), amikacin (2\%) and meropenem (2\%).

Most Enterobacteriacae isolates (94.7\%) had a MAR index higher than 0.2, while the MDR among NFB was lower (31.4\%) (Table 5).
Table 1. Distribution of bacterial isolates from selected poultry farms at three locations in Gaza.

\begin{tabular}{|l|c|c|c|c|c|c|c|}
\hline \multirow{2}{*}{$\begin{array}{l}\text { Gram Negative } \\
\text { isolates }\end{array}$} & Total & \multicolumn{6}{|c|}{ Governorate } \\
\cline { 3 - 9 } & & Gaza & $\begin{array}{c}\text { South } \\
\text { Gaza }\end{array}$ & \multicolumn{2}{|c|}{$\begin{array}{c}\text { The } \\
\text { Middle }\end{array}$} \\
\hline & 360 & 158 & 43.9 & 45 & 12.5 & 157 & 43.6 \\
\hline $\begin{array}{l}\text { Enterobacteriacae } \\
\text { Non-Fermenters } \\
\text { (Pseudomonas sp.) }\end{array}$ & 56 & 0 & 0 & 24 & 42.9 & 32 & 57.1 \\
\hline Total & 416 & 158 & 38 & 69 & 16.6 & 189 & 45.4 \\
\hline
\end{tabular}

Figure 1: Percentage of Enterobacteriacae species isolated from poultry meat and cloacal swabs.

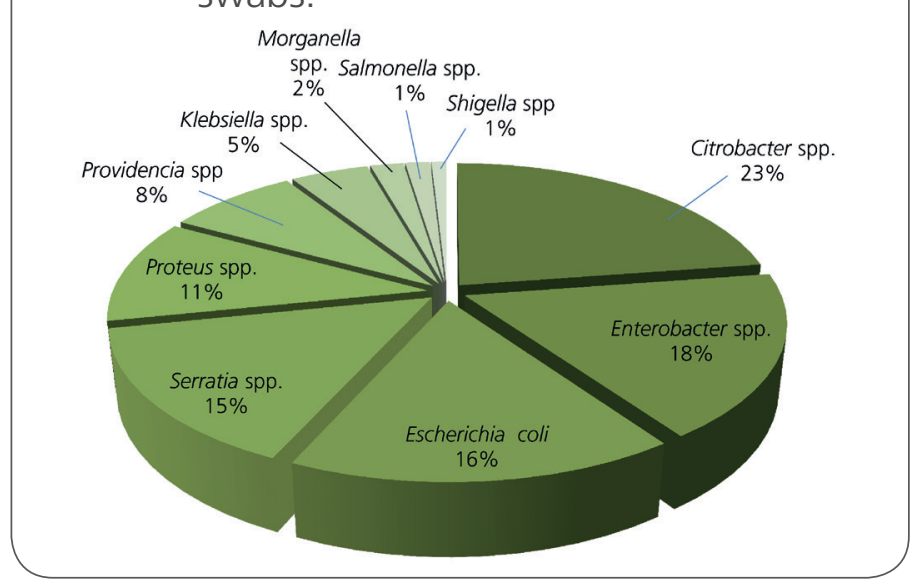

Table 2. Over all resistance rates of Gram-negative bacterial isolates to the tested antimicrobial agents.

\begin{tabular}{|l|c|c|c|}
\hline \multirow{1}{*}{ Antimicrobial } & No. & \multicolumn{2}{c|}{ Resistance } \\
\cline { 2 - 4 } & & $\mathrm{N}$ & $\%$ \\
\hline Amikacin & 338 & 1 & 0.3 \\
\hline Ampicillin & 316 & 270 & 85.4 \\
\hline Ceftazidime & 340 & 150 & 44.1 \\
\hline Ceftriaxone & 292 & 90 & 30.8 \\
\hline Cefuroxime & 324 & 74 & 22.8 \\
\hline Chloramphenicol & 331 & 245 & 74 \\
\hline Ciprofloxacin & 338 & 160 & 47.3 \\
\hline Gentamicin & 341 & 160 & 46.9 \\
\hline Imipenem & 335 & 174 & 51.9 \\
\hline Meropenem & 339 & 6 & 1.8 \\
\hline Nitrofurantoin & 280 & 132 & 47.1 \\
\hline Tetracycline & 324 & 305 & 94.1 \\
\hline TM/SXT & 322 & 258 & 80.1 \\
\hline
\end{tabular}

TM/SXT: Trimethoprim/Sulfamethoxazole. 
Table 3. Distribution percentage of antimicrobials resistance among Enterobacteriacae species.

\begin{tabular}{|c|c|c|c|c|c|c|c|c|c|c|}
\hline \multirow[b]{3}{*}{ Antimicrobial } & \multicolumn{10}{|c|}{ Resistance of Enterobacteriacae isolates } \\
\hline & \multicolumn{10}{|c|}{ No. $/ \%$} \\
\hline & $\begin{array}{l}\frac{\pi}{0} \\
\frac{0}{0} \\
\frac{2}{2} \\
\frac{0}{2} \\
\frac{0}{2} \\
\frac{0}{2}\end{array}$ & 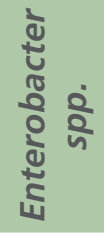 & 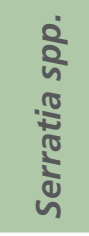 & 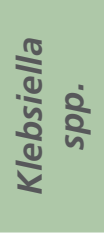 & 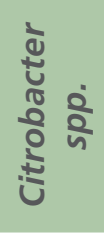 & $\begin{array}{l}\frac{0}{2} \\
n \\
n \\
0 \\
0 \\
0 \\
0 \\
0\end{array}$ & $\begin{array}{l}\text { ¿े } \\
\text { uं }\end{array}$ & $\begin{array}{l}\stackrel{0}{0} \\
\frac{0}{0} \\
\frac{0}{0} \\
\frac{0}{2} \\
\vdots \\
\Sigma\end{array}$ & 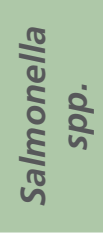 & $\begin{array}{l}\frac{2}{2} \\
\frac{0}{2} \\
\frac{0}{2} \\
\frac{0}{5}\end{array}$ \\
\hline Antimicrobial & 27 & 60 & 51 & 16 & 77 & 38 & 56 & 7 & 3 & 3 \\
\hline Amikacin & 0 & 0 & 0 & 0 & 0 & 0 & 1 & 0 & 0 & 0 \\
\hline Ampicillin & 9 & 19.5 & 13.9 & 5.3 & 20.7 & 9.4 & 19.1 & 1.9 & 0.8 & 0.8 \\
\hline Ceftazidime & 8.8 & 17.4 & 14.8 & 6 & 18.8 & 14.1 & 15.4 & 2.7 & 0.7 & 0 \\
\hline Ceftriaxone & 11.3 & 19.3 & 17 & 5.7 & 21.6 & 9.1 & 10.2 & 4.5 & 0 & 1.1 \\
\hline Cefuroxime & 13.9 & 19.4 & 5.6 & 7 & 27.8 & 13.9 & 8.3 & 2.8 & 0 & 1.4 \\
\hline Chloramphenicol & 8.3 & 19.2 & 15 & 5.5 & 20.5 & 8.7 & 18.3 & 2.5 & 0.4 & 0.8 \\
\hline Ciprofloxacin & 7 & 24.7 & 12 & 5.7 & 23.4 & 4.4 & 20.3 & 1.3 & 0 & 0.6 \\
\hline Gentamicin & 10 & 21.3 & 8.1 & 8.1 & 22.6 & 6.3 & 20 & 2.5 & 0 & 1.3 \\
\hline Imipenem & 7.1 & 11.7 & 18.7 & 6.5 & 21.7 & 16.4 & 13.5 & 2.3 & 1.2 & 0.6 \\
\hline Meropenem & 33.3 & 16.7 & 16.7 & 0 & 0 & 16.7 & 16.7 & 0 & 0 & 0 \\
\hline Nitrofurantoin & 5.3 & 11.4 & 21.4 & 7.6 & 17.6 & 20.6 & 11.5 & 3.1 & 0.8 & 0 \\
\hline Tetracycline & 7.4 & 18.8 & 15.1 & 4.7 & 21.8 & 11 & 16.7 & 1.7 & 1 & 1 \\
\hline $\begin{array}{l}\text { Trimethoprim/ } \\
\text { Sulfamethoxazole }\end{array}$ & 7.1 & 20.1 & 15.7 & 5.1 & 23.6 & 9.5 & 14.6 & 2.8 & 0.8 & 0 \\
\hline
\end{tabular}

Table 4. Distribution of resistance rates among Gram negative non-fermenting bacilli bacterial isolates $(\mathrm{N}=51)$ to tested antimicrobial agents.

\begin{tabular}{|l|c|c|}
\hline \multicolumn{1}{|c|}{ Antimicrobial } & Isolates & Resistance \\
\hline Amikacin & N & $\%$ \\
\hline Azithromycin & 1 & 2 \\
\hline Cefepime & 0 & 0 \\
\hline Ceftazidime & 2 & 3.9 \\
\hline Gentamicin & 11 & 21.6 \\
\hline Imipenem & 15 & 32.6 \\
\hline Levofloxacin & 6 & 11.8 \\
\hline Meropenem & 13 & 25.5 \\
\hline
\end{tabular}

Table 5. Multi drug resistance in Gram-negative isolates.

\begin{tabular}{|l|c|c|}
\hline \multirow{2}{*}{ Valid } & Gram negative bacilli & $\begin{array}{c}\text { Gram negative non- } \\
\text { fermenting bacilli }\end{array}$ \\
\cline { 2 - 3 } & $\%$ & $\%$ \\
\hline MDR & 94.7 & 31.4 \\
\hline Non & 5.3 & 68.6 \\
MDR & 100 & 100 \\
\hline Total & &
\end{tabular}

\section{Discussion}

The World Health Organization (WHO) has recognized that antimicrobial resistance is a global problem that calls for a global response. Consequently, WHO issued the global principles for the containment of antimicrobial resistance in animals intended for food. The WHO global strategy for the containment of 
antimicrobial resistance where some interventions were recommended that hopefully will enable local authorities to slow down the emergence and reduce the spread of resistance in diverse settings [14]. Transfer of resistant bacteria between animals and humans through food products has been documented and can pose a threat to public health [15].

This study has attempted to determine the resistance patterns of Gram-negative bacteria isolated from poultry in Gaza, Palestine. The overall percentage of E. coli among Enterobacteriaceaewas 16\%, this is low when compared to the prevalence rate of $37.86 \%$ obtained by Rahman et al.; [16] and $21.09 \%$ by Rahman et al., [17]. Even very much higher results were reported with a prevalence rate of $76 \%$ by Barua et al., [18], and (78.9\%) by Jakaria et al., [19].

Antimicrobial resistance among Enterobacteriaceae and NFB isolates was determined against 16 commonly used antibiotics. Although the pathogenicity of these isolates was not examined, the genes responsible for multi-drug resistance may be transmitted to people by food and may results in serious public health hazards.

In our study, only one isolate of $E$. coli was resistant to amikacin, this is not comparable to the prevalence rate of $37.7 \%$ obtained by Rahman et al. [16] and differ greatly from the findings of Arya et al. (2008) who reported that $80 \%$ of Shiga toxin producing $E$. coli (STEC) strains were resistant to amikacin, tetracycline(63\%), and ciprofloxacin (20\%) [20].

The efficacy of tetracycline against Enterobacteriaceae isolates was shown to be the least effective as evident by the high resistance percentages (94.1\%). This result is close to the results obtained in Indonesia by Yulistiani et al. (69.9\%) [21] and very much higher than the percentage reported by Azevedo et al. (52.38\%) [22]. High resistance to tetracycline in this study can be interpreted by the extensive, uncontrolled, widely and long use of tetracycline in poultry farms as a growth promotor agent, and for disease prevention. Improper and intensive use of antibiotics in poultry may be one trigger of antibiotic resistance particularly to antibiotics that are often used on the chicken farms. Bacteria can become resistant to tetracycline by at least three mechanisms; enzymatic inactivation of tetracycline where an acetyl group is added to the molecule causing inactivation of the drug, efflux resistance gene encodes a membrane protein that actively pumps tetracycline out of the cell, and ribosomal protection which blocking tetracycline from binding to the ribosome $[23,24]$.

Our results showed that $94.7 \%$ and $31.4 \%$ of the tested Enterobacteriacae and NFB isolates were MDR. The high prevalence of antimicrobial resistance obtained can be explained by the common practice of using of antimicrobials as prophylactics and growth enhancers in poultry production. Unfortunately, the authors were not able to find any published data regarding the amount of antimicrobials usage in Gaza, however, one study from Gaza strip reported malpractices and abuse of antimicrobials in poultry farms [25].

The result of the antibiotics resistance patterns for Enterobacteriaceae obtained by Yulistiani et al. [21], showed high level of multi-drug resistant bacteria, yet lower than obtained in this study. As many as $61.1 \%$ of Enterobacteriaceae isolates were MDR to at least three unrelated antimicrobial agents.

In our study, the dominant resistance was to tetracycline, and ampicillin in $94.1 \%, 85.4 \%$, respectively. This result is higher than the results obtained by Azevedo et al. who reported resistance of $32.8 \%$ for Ampicillin and only 4\% for tetracycline [22].

Carbapenem-resistant Enterobacteriaceae (CRE) isolates are a serious public health issue due to limited therapeutic options to treat such infections. CRE isolates have been predominantly isolated from humans and environmental samples and they are rarely reported among isolates from poultry [26].

Meropenem resistance among all isolates was low (1.8\%) while higher resistance against imipe- 
nem was demonstrated (51.9\%). These results are similar to those obtained by Webb et. al., who investigated the susceptibility of the bacterial isolates to the carbapenems including imipenem, and meropenem, and showed that out of the 360 Enterobacteriacae isolates recovered, $51.9 \%$ and $1.8 \%$ were resistant to imipenem, and meropenem, respectively [27]. Based on the results described herein, CRE appear to be high among chicken.

Recent studies have highlighted the global emergence of CRE isolates in both livestock [28] and companion animals [29]. In particular, recent studies demonstrating the isolation of carbapenemase-producing E. coli (NDM-1 and OXA-48) and Klebsiella pneumoniae (OXA-48) from clinical infections in dogs [29] and questions about the veterinary use of carbapenems are raised [30]. Carbapenemase producing organisms were isolated from 5 of 127 (4\%) products tested [31].

This study shows that both Salmonella and Shigella isolated from poultry samples were highly susceptibility to almost all tested agents and none of them was resistant to meropenem.

A study conducted in one dairy farm in France, 2010, samples taken from 50 dairy cows were tested. Nine of the samples were positive for carbapenamse producing (CP) in Acinetobacter spp. isolates [32]. While a survey on chicken and duck farms and pig slaughterhouse in Eastern China showed that nine isolates from different bacterial genera (Stenotrophomonas, Myroides, Chryseobacterium, and Acinetobacter) were non-susceptible to carbapenems [28]. From longitudinal studies conducted in swine and broiler farms in Germany [33], two fattening pig farms and broiler farm were found to be positive for carbapenamse producing Salmonella spp. One of these farms was also positive for CP E. coli.

Carbapenems are not licensed for use in foodproducing animals in Europe and other parts of the world. Therefore, one simple and effective control approach to minimize the further emergence and possible spread of carbapenem-resistant strains via the food chain would be to continue to ban the use of carbapenems in food-producing animals [34]. There are no reports of using carbapenems in poultry in Gaza, and one explanation for this high rate of carbapenem resistance among poultry isolates is that human strains may have colonized those poultry farms. This result can be supported by the fact that many poultry farms are found in residential areas and there are no infection control measures practiced by poultry farmers.

In conclusion, MDR among Gram-negative bacteria isolated from chicken cloacal swabs and chicken meat was found in high rates in Gaza. Carbapenem resistance was also prominent most especially for imipenem. This result requires further investigation to track the source of carbapenem-resistant bacteria. The Ministry of Agriculture in Gaza should implement monitoring programs and control measures to combat this serious public health issue.

\section{Acknowledgements}

The authors would like to acknowledge the Advisory Group on Integrated Surveillance of Antimicrobial Resistance (AGISAR) for funding this research project and the Islamic University of Gaza for opening their facilities.

\section{References}

1. Rabinowitz PM and Conti LA, Human-Animal Medicine-E-Book: Clinical Approaches to Zoonoses, Toxicants and other Shared Health Risks. 2009: Elsevier Health Sciences.

2. Rwego IB, Isabirye-Basuta G, Gillespie TR and Goldberg TL. Gastrointestinal bacterialtransmission among humans, mountain gorillas, and livestock in Bwindi Impenetrable National Park, Uganda. Conserv Biol 2008; 22(6): 1600-1607.

3. Middleton $J$ and Ambrose A. Enumeration and antibiotic resistance patterns of fecal indicator organisms isolated from migratory Canada geese (Branta canadensis). J Wildl Dis 2005; 41(2): 334-341. 
4. Coque TM, Novais Â, Carattoli A, Poirel L, Pitout J, et al. Dissemination of clonally related Escherichia coli strains expressing extended-spectrum $\beta$-lactamase CTX-M-15. Emerg Infect Dis 2008; 14(2): 195.

5. Martel J-L, Tardy F, Sanders $P$ and Boisseau J. New trends in regulatory rules and surveillance of antimicrobial resistance in bacteria of animal origin. Vet Res 2001; 32(3-4): 381-392.

6. Silva N, Igrejas G, Figueiredo N, Gonçalves A, Radhouani $H$, et al. Molecular characterization of antimicrobial resistance in enterococci and Escherichia coli isolates from European wild rabbit (Oryctolagus cuniculus). Sci. Total Environ. 2010; 408(20): 4871-4876.

7. Köhler M. OIE. Manual of standards for diagnostic tests and vccines, Office International des Epizooties, Washington DC, USA (1997), XXXII, 723 pages, 1998; Urban \& Fischer.

8. Astal Z, El-Manama A and Sharif F. Antibiotic resistance of bacteria associated with community-acquired urinary tract infections in the southern area of the Gaza Strip. J Chemother 2002; 14(3): 259-264.

9. Elmanama $\mathrm{AA}$ and Abdelateef $\mathrm{N}$. Antimicrobial resistance of enteric pathogens isolated from acutegastroenteritis patients in Gaza strip, Palestine. IAJAA 2013; 2(4).

10. Elmanama AA. Vancomycin resistant enterococci (VRE) among Non-Hospitalized Individuals in Gaza City, Palestine. Age 2008; 1: $17-40$

11. Krumperman PH. Multiple antibiotic resistance indexing of Escherichia coli to identify high-risk sources of fecal contamination of foods. Appl Environ Microbiol 1983; 46(1): 165-170.

12. CLSI. Performance standards for antimicrobial susceptibility testing. Approved Standards CLSI. 2010: M100-S20.

13. Sandhu R, Dahiya $S$ and Sayal P. Evaluation of multiple antibiotic resistance (MAR) index and Doxycycline susceptibility of Acinetobacter species among inpatients. Int J Infect Dis 2016; 45: 327.

14. World Health Organization. WHO global strategy for containment of antimicrobial resistance: executive summary. 2001; Geneva: World Health Organization.

15. Akinjogunla $O$, Inyang $C$ and Akinjogunla $V$. Bacterial species associated with anatomical parts of fresh and smoked Bonga Fish (Ethmalosa fimbriata): Prevalence and Susceptibility to Cephalosporins. Res J Microbiol 2011; 6(1): 87-97.

16. Rahman M, Rahman A, Islam M and Alam M. Antimicrobial resistance of Escherichia coli isolated from milk, beef and chicken meat in Bangladish. Bangladesh J Veterin Med 2017; 15(2): 141-146.

17. Rahman M, Samad M, Rahman $M$ and Kabir S. Bacteriopathological studies on salmonellosis, colibacillosis and pasteurellosis in natural and experimental infections in chickens. Bangladesh J Veterin Med 2004; 2(1): 1-8.
18. Barua R, Bhilegaonkar K, Agarwal R, Singh D, Malik S, et al. Prevalence and rapid detection of shiga toxin producing $\mathrm{E}$. coli (STEC) in water and ready-to-eat (RTE) foods. Journal of Veterinary Public Health. 2007; 5(2): 75-83.

19. Jakaria A, Islam MA and Khatun MM. Prevalence, characteristics and antibiogram profiles of Escherichia coli isolated from apparently healthy chickens in Mymensingh, Bangladesh. Microbes \& Health. 2012; 1(1): 27-29.

20. Arya G, Roy A, Choudhary V, Yadav MM and Joshi C. Serogroups, atypical biochemical characters, colicinogeny and antibiotic resistance pattern of Shiga toxin囚producing Escherichia coli isolated from diarrhoeic calves in Gujarat, India. Zoonoses Public Health. 2008; 55(2): 89-98.

21. Yulistiani R, Praseptiangga D, Raharjo D and Shirakawa T. Prevalence of antibiotic-resistance Enterobacteriaceae strains isolated from chicken meat at traditional markets in Surabaya, Indonesia. in IOP Conference Series: Materials Science and Engineering. 2017. IOP Publishing.

22. Azevedo I, Albano H, Silva J and Teixeira P. Antibiotic resistance of Enterobacteriaceae isolated from the domestic food related environments. Journal food quality and hazards control 2015; 2(2): $51-55$

23. Alsan M, Schoemaker L, Eggleston K, Kammili N, Kolli $P$, et al. Out-of-pocket health expenditures and antimicrobial resistance in low-income and middle-income countries: an economic analysis. The Lancet infect dis 2015; 15(10): 1203-1210.

24. Nguyen F, Starosta AL, Arenz S, Sohmen D, Dönhöfer A, et al. Tetracycline antibiotics and resistance mechanisms. Biol Chem 2014; 395(5): 559-575.

25. Elmanama AA and Albayoumi MA. High prevalence of antibiotic residues among broiler chickens in Gaza strip. Food and Pub Health 2016; 6(4): 93-98.

26. Abraham S, O'dea M, Trott DJ, Abraham RJ, Hughes D, et al. Isolation and plasmid characterization of carbapenemase (IMP4) producing Salmonella enterica Typhimurium from cats. Sci. Rep. 2016; 6: 35527.

27. Webb HE, Bugarel M, Den Bakker HC, Nightingale KK, Granier $\mathrm{SA}$, et al. Carbapenem-resistant bacteria recovered from faeces of dairy cattle in the high plains region of the USA. PLoS One 2016; 11(1): e0147363

28. Wang Y, Wu C, Zhang Q, Qi J, Liu H, et al. Identification of New

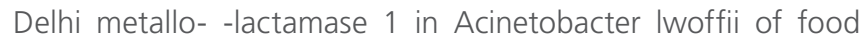
animal origin. PLoS One 2012; 7(5): e37152.

29. Stolle I, Prenger-Berninghoff E, Stamm I, Scheufen S, Hassdenteufel E, et al. Emergence of OXA-48 carbapenemaseproducing Escherichiacoli and Klebsiellapneumoniae in dogs. J Antimicrob Chemother 2013; 68(12): 2802-2808. 
30. Abraham S, Wong HS, Turnidge J, Johnson JR and Trott DJ. Carbapenemase-producing bacteria in companion animals: a public health concern on the horizon. J. Antimicrob Chemother 2014; 69(5): 1155-1157.

31. Rubin JE, Ekanayake $S$ and Fernando C. Carbapenemaseproducing organism in food, 2014. Emerg Infect Dis 2014; 20(7), 1264-1265

32. Poirel $L$, Berçot $B$, Millemann $Y$, Bonnin RA, Pannaux $G$, et al. Carbapenemase-producing Acinetobacter spp. in cattle, France. Emerg Infect Dis 2012; 18(3): 523.

33. Laube H, Friese A, Von Salviati C, Guerra B, Käsbohrer A, et al. Longitudinal monitoring of Esbl/Ampc-Producing Escherichia coliin German broiler chicken fattening farms. Appl Environ Microbiol 2013; AEM, 00856-13. DOI: 10.1128

34. Hazards EPOB. Scientific Opinion on Carbapenem resistance in food animal ecosystems. First published in the EFSA Journal: 17 December 2013.

Publish in International Archives of Medicine

International Archives of Medicine is an open access journal publishing articles encompassing all aspects of medical science and clinical practice. IAM is considered a megajournal with independent sections on all areas of medicine. IAM is a really international journal with authors and board members from all around the world. The journal is widely indexed and classified Q2 in category Medicine. 